handlung wies Ballot weiter nach, dass der kălteren Seite der Sonne, die $1846^{\circ}$ 1. Jan. uns gegenüberstand, eine durchschnittlich $\mathrm{nm} 0^{07} \mathrm{C}$. niedrigere Temperatar entsprach, als der wärmeren, die $1846 \mathrm{Jan}, 15$ uns gegenüberstand. Carlini u. d'A rrest fanden nahezu dasselbe Resultat wie Nervander, Airy dagegen konnte aus Greenwicher Beobachtungen dasselbe nicht entscheiden. Nachdem nun durch die ausgezeichneten Arbeiten des Herrn Prof. C. Hornstein, Directors der Sternwarte in Prag und J. Broun nachgewiesen ist, dass die Rotationszeit der Sonne aus den Veränderungen der magnetischen und barometrischen Erscheinungen sich viel genauer und das aus einer $k u r z e n$ (1 Jahr) Reihe von Beobachtungen ableiten lässt, und da sowohl die Periode von Nervander wie die von Ballot so sehr von der Spoerer'schen und Carrington'schen Rotationsperiode abweicht, unterzog ich die Prager Temperaturbeobachtungen des Jabres 1876 einer näleren Untersuchung, eine viel kürzere Periode aus diesen Beobachtungen erwartend, als Herr Dir. Hornstein aus magn. und barom. Beobachtungen erhielt, da ich mir vorstellte, dass sie der aus Fleckenbeobachtungen abgeleiteten Rotation des thermischen Aequators der Sonne, der bekanntlich mit dem wirklichen Aequator der Sonne nicht zusammenfallt, entsprechen wärde. Ich bearbeitete des- balb die Beobachtungen nach der von H. Dir. Hornstein in den Sitzungsberichten der Wiener k. k. Akademie (Bd. 67) gegebenen Methode, die sich zu solchen Untersuchungen insbesondere eignet. Ich theile hier das Schlussresultat mit. Es ergab sich ein wahrscheinlichster Werth von $T=25.56$ Tage. Nach Carrington's Beobachtungen würde sich nach obiger Auffassung für die Lage des thermischen Aequators der Sonne die Breite von $10^{\circ}$ bis $20^{\circ}$ ergeben, nach Spoerer's Beobachtungen die Breite ron 13 bis $14^{*}$.

$\mathrm{Da}$ nach neueren Untersuchungen der Einfluss der Peränderung der Krätte der Sonne sich in den Ver. anderungen der meteorologischen Erscheinungen abspiegelt, untersuchte ich ferner die Wind-Componenten des Jahres 1870 in ihrer Beziehung zur Rotationszeit der Sonne. Für die Ost-West-Componenten fand ich eine Periode ron 26.71 Tagen. $\mathrm{Ob}$ aus diesem Resultate auf eine Correspondenz zwischen herrschenden Winden auf der Sonne, die Spoerer aus seinen Fleckenbeobachtongen abgeleitet hat, za sehliessen wäre, wird eine nähere Untersuchung entscheiden.

IVien, 4. December 1878.

$$
\begin{gathered}
\text { Dr. G. Gruss. } \\
\text { Assistent }
\end{gathered}
$$

am k. k. Polytechn.

\title{
Comet II 1873 (Tempel).
}

Zu meiner Beobachtung des Cometen, $187 \mathrm{~s}$ Oct. 15, ist nachträglich zu bernerken, dass, wie mir Herr Schulhof gefälligst meldet, der Vergleichstern sich in den Wash. Zones findet. Vergl. Tempel in Astr. Nachr. Nr. 2229 bei October 16 , wo derselbe Vergleichstern vorkommt.

Am 15ten November habe ich nochmals autmerksam die Gegend, wo der Comet nach Schulhofs Ephemeride stehen müsste, durchsucht; es ward aber der Comet nicht mehr gefunden.

Athen 187 is Dec. 3.

J. F. Julius Schmidt,

\section{Berichtigung zu Bruhns' Logarithmentafeln,}

Some time ago I detected the following error in Brubns' Manual of Logarithms (Stereotype Edition 1870.) p. 209 - Tan. $0322^{\prime} 27^{\prime}$ Diff. for 2330 read 2230 . As this error does not seem to have been corrected in the last Edition (Second Sterentype 135s) which I have just obtained - it is possible that it has eseaped notice.

Thomas Wright.

Inhalt:

Zn Nr. 2238. C. Bruhns. Planetenbeobachtungen. 81. - Wilh. Tempel. Beobachtungen des Cometen II 1873. 91. - G. Gruss. Beiträge zur physischen Beschaffenheit der Sonne. 93. - F. F. Fulius Schmidt. Comet II 1873 (Tempel). 95. - Thomas Wright. Berichtigung zu Bruhns' Logarithmentafeln. 95. 